the next day they get up for two similar periods, and are told that they may walk to the lavatory if they want to. Not many avail themselves of this permission until the third day. From that time they are told that they may do anything that they can do, and are up the greater part of the day. Many play billiards, help with light ward duties, and walk about the grounds. They are given no specific exercises until the eighth day, when they are transferred to J.W.O. Convalescent Hospital, and begin graduated physical training with a sergeant instructor. An interesting side-light has come from the nursing staff, who are able to predict with a fair degree of accuracy which patients will have post-operative respiratory complications before operation has taken place. They have noted that the anxious, miserable, worried, unhappy patients who are afraid of hurting themselves will get respiratory complications, while the happy active type will succeed in avoiding them. This point has also been noted by Lucas (1944). If it should be thought that this regime is unduly rigorous the details of the recovery of my daughter, who had a simple herniotomy done, may be of interest. She followed this routine exactly, and had been told from the outset that she might do anything that she was able to do so long as it did not hurt. She got out of bed readily after 24 hours and was walking with confidence on the third day. She left hospital on the eighth day and travelled by train across Britain. From that time she was running about with her brothers and swimming from a beach one and a half miles from home. On the fourteenth day she turned six cartwheels in succession without any hesitation or thought of having recently had an operation. She went back to school without any complaint.

It is notoriously difficult to follow up Service cases, but a questionary has been sent out to 200 consecutive cases one year after operation, and reports have been received from 123 . Among these there have been 8 recurrences-i.e., $6.5 \%$. Among the remainder, information is given that many of them are serving over-seas, and it may be assumed that recurrences had not occurred among these men-at any rate before they sailed. Of the rest it is fair to assume that those who have a recurrence or are in any way disabled are more likely to answer questions than those who are quite well. Answers have been received from every battlefield where British troops are engaged. It would appear that a routine of early activity has not increased the recurrence rate.

\section{Summary of Conclusions}

1. The rate of recurrence of operations for inguinal hernia is higher than is often realized.

2. Early activity is not a factor leading to recurrence.

My thanks are due to the Director-General, E.M.S., for permission to publish this report.

REFERENCES

Lucas, B. G. B. (1944). Proc. roy. Soc. Med., 37, 145.
Maingot, R. (1941). British Medical Journal, 1, 777.
Martin, D. J., et al. (1943). Ibid., 1, 754.
Page, C. M. (1943). Proc. roy. Soc. Med., 36, 185.
Tanner. N. C. (1942). Brit. J. Surg., 29, 285.

\section{Medical Memoranda}

\section{A Case of Locked Twins}

The following case of locked twins appears to be unusual enough to warrant publication.

The patient, a secundipara aged 30 , was seen by me several times during her pregnancy for routine antenatal examination. Her last normal menstrual period was from May 24 to 28,1943 , but she had a further period of vaginal bleeding from July 18 to 22 before she consulted me. This made it difficult to estimate the correct date for the expected confinement, but according to the size of the uterus it appeared that she was due to be confined about March 15-i.e., four weeks earlier than the actual labour. At my last consultation on the expected date, March 15, I informed the patient of the possibility of twins, owing to the large size of the abdomen and the difficulty of ascertaining the foetal position.

On April 17 at 3.30 a.m. the waters broke, and at 9.30 a.m. she started getting weak pains. Towards 7 p.m. the midwife made a rectal examination and thought she felt a foot presenting through the fully dilated cervix. I confirmed this finding. with a further rectal examination at 7.15 p.m., and, to the right of the foot, felt what I took to be the half-breech. The patient-was bearing down strongly with good five-minute pains. On abdominal examination no definite positions could be distinguished, as foetal parts could be felt everywhere, and in the left flank there was an ovoid mass which the midwife insisted was a head, but this was subsequently disproved. One foetal heart was heard in the right flank below the umbilicus, and another in the left flank at the level of the umbilicus. The difference in rate between the two was not enough to be significant. A diagnosis of twins was made, and I anticipated no trouble. However, after about an hour the pains became weaker and less frequent, and there was no apparent progress. The patient showed signs of exhaustion, and at about 9 p.m. I decided to makc a vaginal examination to find out what was holding up the progress of labour. I found a leg lying in the vagina, and could feel along the leg as far as the mid-thigh; but, to my surprise, lying to right of the leg was a head covered by a bag of membranes, and it was apparent that the head of the one twin and the half-breech of the other had entered the pelvis together and become locked. What I had taken for the buttock, on rectal examination, was now disclosed as the second head covered by a " bag of waters." The latter bag of membranes, incidentally, was formed by a fold of membrane being caught between the two twins, as we subsequently found only one placenta and chorion.

I sent for the aid of Dr. David Gamsu, and under deep ether anaesthesia he pushed the presenting head up out of the pelvis with his vaginal hand and held it up with his external hand. We thereupon pulled the half-breech of the other twin down and delivered this twin as a breech presentation. The infant was a female weighing $5 \mathrm{lb}, 3 \mathrm{oz}$. and, like its sister, exhibited only slight blue asphyxia. Delivery was complete at about 10.30 p.m., a single placenta being born about 30 minutes after the second foetus, and the post-partum haemorrhage was normal in amount.

The puerperium was normal, and the patient got up on the 10th day, both babies doing very well. The case appears to be interesting because of $(a)$ the comparative rarity of locked twins-the usual incidence given being 1 in 90,000 pregnancies; $(b)$ the unusual method of locking.

I am indebted to Dr. D. Gamsu for the delivery of a difficult case and his help with these notes.

Johannesburg, S. Africa. $\quad$ B. A. Bradlow, M.B., B.Ch.

\section{Quinsy, complicated by Deep Pharyngeal Throm- bosis, Multiple Infarcts of the Lungs, and Myocarditis}

Quinsy appears to be a fairly common complaint among Service personnel, but the complications in this case are unusual and should be of interest to readers. I am unable to find a similar case in the literature.

\section{CASE ReCORD}

On July 15, 1943, a W.A.A.F. was admitted to hospital as a case of diphtheria. On the previous day she was admitted to the R.A.F sick bay complaining of nausea, vomiting, sore throat, and general malaise; temperature $101^{\circ}$, pulse 84 . The throat was inflamed and there was exudate on both tonsils. The temperature rose to $105^{\circ}$ despite tepid sponging.

On admission the patient was ill and extremely pale. Examination showed: no oral fetor; right cervical adenitis and right tonsillar gland acutely tender; throat very inflamed, with gross oedema of right tonsil; right anterior faucial pillar pushed well forward; left tonsil much less involved; no membrane; stream of pus down post-pharyngeal wall coming from the right tonsil. The condition was characteristic of quinsy with good drainage. Swlabanilamide started. Patient developed a swinging temperature, $102^{\circ}$ $104^{\circ}$ to $97^{\circ}$; trismus. Pulse septicaemic in type.

July 17. - Despite the fact that the throat condition improved, the patient became much worse and developed a fine tremor of the left leg.

July 19.-Pulse septicaemic-very full and bounding. Extremely toxic; rigors. Temperature $104^{\circ}$, but throat condition improved and oedema well reduced. Patient's manner very strange. Resistant to examination. Quinsy still draining. W.B.C., 9,884 per c.mm. On July 20 sulphanilamide was stopped : total $14 \mathrm{~g}$.

July 21. - Condition deteriorated. Rigors; profuse sweats; quinsy almost resolved, but temperature continued to swing $-102^{\circ}, 104^{\circ}$

Tremor of left leg continued. Liver palpable $1 / 2$ in. below costal margin. W.B.C., 29,952. A radiograph of the chest revealed some fluffy densities in the right upper zone; the right diaphragm was raised and flat, and the illumination of the right lower zone was poor. Diagnosis: thrombosis of pharyngeal plexus involving the veins close to the internal jugular vein. Sutphapyridine begun.

July 23.-Blood culture positive for non-haemolytic streptococci. Patient complained of pain in right side of neck. A tender swelling felt in the region of the right jugular vein. W.B.C., 23,000

July 26.- Large tender mass palpable along right jugular vein just above the level of the tonsillar gland. Palatal and right tonsillar oedema; stream of pus coming from the old quinsy opening. Nex day a transfusion of one pint of fresh blood was given. Sulphapyridine was stopped on July 29: total $40 \mathrm{~g}$.

July 30.-Acute left-sided pleurisy. Dyspnoea well marked; very slight haemoptysis. The neck condition improved-not tender; hard knotty mass about $3 / 4$ in. long palpable along the jugular chain. Quinsy dry. W.B.C., 14,040. A course of sulphadiazine started on Aug. 2.

Aug. 3.-Dry unproductive cough with pain in the left chest. Crepitations ++ left lower lobe. Throat tender; some pus again leaking from quinsy. $X$ rays showed massive density in the right upper zone laterally and densities in the right middle and lower zones. The appearances were not like a tuberculous lesion and were compatible with infarcts.

Aug. 4.-Sulphadiazine stopped: total $25 \mathrm{~g}$. Sulphapyridine 30 the patient had three attacks of pleural rub. 
Sept. 27.-Mild laryngitis. Streaks of pus on post-pharyngeal wall. Three days later the throat condition cleared up completely. Oct. 5.-Radiographs of chest showed previous densities completely cleared up. The right. diaphragm was still raised and flat, but apart from this there was nothing abnormal.

The patient was discharged on Oct. 15 for three months' rest. The myocarditis was still present, but had improved. The total amount of sulphonamides given was $103 \mathrm{~g}$. over a period of 26 days. The only toxic symptom was vomiting. These drugs were discontinued when the white count fell to 12,000 .

\section{COMMENT}

In this case of quinsy, venous thrombosis resulted in suppuration in the deep pharyngeal plexus, which eventually drained back into the pharynx through the original thrombosed vessel This was demonstrated by the fact that light pressure produced green pus via the superior tonsillar fossa. Further complications resulted in extensive relatively sterile thrombosis throughout almost the whole of the cervical portions of the internal jugular vein.

It is suggested that the rigors resulted from almost sterile pieces of clot and that the ensuing metastases produced sterile pulmonary infarcts.

My thanks are due to Dr. George Hurrell, medical superintendent, City Hospital for Infectious Diseases, Newcastle-upon-Tyne, for interpretation o Newcastle-upon-Tyne, for permission to publish this case.

\section{Walker Gate}

Newcastle-upon-Tyne.

\section{R. R. J. R. Clarke, M.B., B.Ch.} Resident Medical Assistant City Hospital for Infectious Diseases.

\section{Purpura Fulminans}

The following account of a case of purpura fulminans is thought to be of interest because of the rarity of the condition.

\section{CASE Report}

The patient, a girl aged $6 \frac{1}{2}$, was admitted in a stuporous condition on June 25,1944 , with a diagnosis of acute purpura. Two days previously she had developed purpuric spots, but had felt perfectly well, her only complaint being a slight headache on the night before admission. She had had no recent illness. On the morning of admission her mother had suddenly found her unconscious.

On examination the patient was very restless and almost unconscious. The temperature per rectum was subnormal and the pulse was raised only during bouts of particular restlessness. -There were large symmetrical purpuric ecchymoses on the arms and legs and a few on the trunk. A very few minute spots were present in the buccal cavity, which was examined with difficulty owing to a tightly clenched jaw. The pupils were then equal and the retinae showed gross haemorrhages, especially on the left side. There was an internal strabismus. Nothing abnormal was found in the chest, but the heart gave a haemic murmur. The abdominal wall was the heart gave a haemic murmur. The abdominal wall was The child resented abdominal palpation, especially over the lef upper quadrant. Nothing abnormal was found in the central or peripheral nervous system except a dorsiflexed plantar response. There was no neck rigidity. Lumbar puncture produced a heavily blood-stained fluid at negligible pressure, and in view of this it was decided that blood transfusion would be of no avail. The bleeding time was 8 minutes and the clotting time was normal. The capillary fragility test was positive.

The patient's condition deteriorated throughout the day and finally a generalized spasticity set in. All reflexes were very brisk, though at no time were the abdominal reflexes present. The left pupil was dilated and Cheyne-Stokes breathing began, with occasional laryngeal spasm. The temperature gradually rose to $103^{\circ}$ just before death The pulse was weak and very irregular, and the patient vomited once or twice, producing mucus with a few streaks of blood. She became incontinent of urine. Nepenthe, 5 minims, was given to control restlessness. At 4.15 the next morning she died.

Post-mortem Examination. - The brain seemed large for a child Post-mortem Examination.- The brain seemed large for a child
of her size, but it was not congested or oedematous. The ventricles were filled with blood and clot, which had burst through the brain substance in the region of the posterior horn of the left latera ventricle. The meninges were normal. The heart was normal. The right lung was healthy, but the left was collapsed and engorged with blood. There was free blood in the left thorax. The stomach showed a patch of submucous haemorrhages, through which perforation had occurred. This was considered to be due to postmortem digestion. The gut revealed a few haemorrhages, and one or two were -present in the bladder mucosa. There was slight congestion in the liver and spleen but apart from this those organs were normal. The kidneys, adrenals, and bone marrow appeared
normal. There were no joint effusions. Microscopical examination of blood and cerebrospinal fluid showed one or two lymphocytes per c.cm. in the latter, but no other abnormalities. Death was due to spontaneous cerebral haemorrhage.

I should be grateful to glean any information from others with experience of this condition.

C. R. OYLER, M.R.C.S., L.R.C.P. House Physician, Worthing Hospital.

\section{Reviews}

\section{LYMPH NODE METASTASES}

Lymph Node Metastases. Incidence and Surgical Treatment in Neoplastic Disease. By G. W. Taylor, M.D., F.A.C.S., and Ira Theodore Nathanson, M.S., M.D. With foreword by S. Warren, M.D. (Pp. 498; illustrated. 42s.). London: Oxford University Press.

Metastasis to neighbouring lymph nodes is such a fundamental feature of most forms of malignant disease that one may perhaps question the wisdom of writing a book which concerns itself exclusively with the secondary lymphatic involvement divorced from the primary lesion. The primary principle in the surgical treatment of malignant disease that the growth itself, the secondary (usually lymphatic) extensions, and the intervening tissues should be removed en masse whenever possible still remains unchallenged. In situations where this is technically impossible the treatment of the glandular involvement has to be accomplished by a separate operative approach. Here there may be some justification for considering the glandular problem as an entity in isolation from the disease as a whole, though even so we feel that anything which tends to divide the conception of a single disease process into two compartments must be fundamentally unsound.

This criticism of the scope of Lymph Node Metastases by Taylor and Nathanson of the Harvard Medical School must of course not be regarded as applying to the actual matter or presentation of the subject, for which we have nothing but praise. A large amount of information has been collected in the book which would otherwise need prolonged search through the literature. Statistical tables compiled from studies of large numbers of cases are numerous and valuable, especially as they include some rather unusual aspects, such as the size of the lymph nodes in relation to metastases, and so on. The uncertainty of palpation as a guide to lymph node involvement is clearly brought out, as is the relation of pathological grading to the incidence of metastasis.

The book is divided into three parts, the first of which deals with general considerations, and gives a review of the normal lymphatic anatomy. Part II is concerned with lymph node metastasis on a regional basis, and Part III with certain of the commoner operative techniques. There are some sixty illustrations of anatomical and surgical details. A valuable volume for the surgeon's reference library.

\section{A BOOK ON ANAESTHESIA}

Practical Anaesthetics for Students, Hospital Residents and Practitioners. By J. Ross MacKenzie, M.D., D.A. (Pp. 130; illustrated. 10s. 6d.)

According to the preface this book is intended to form a foundation on which medical students, hospital residents, and occasional anaesthetists can build a "practice of anaesthesia." Yet in this book of 130 pages and with this title such readers will find a disproportionately large amount of space devoted to splanchnic anaesthesia, helium therapy, and anaesthesia for thoracic surgery-subjects of little interest to any but the experienced anaesthetist. The novice will search hard for detailed guidance on everyday practice, and it avails him little to find a description of unilateral anaesthesia for pneumonectomy, caudal anaesthesia for childbirth, and splanchnic anaesthesia by the abdominal approach. Those who want to know practical details of how to use the Boyle machine, or pass an endotracheal tube, or give a spinal anaesthetic, will be disappointed at the sketchy descriptions. One searches in vain for answers to questions which casualty anaesthetists (often junior M.O.s) ask so frequently, such as how to deal with a man with a bleeding wound in the mouth or a straightforward sucking wound in the chest. Here and there the author touches on physiological or physical points, but some of his statements are open to question. He says, for example, that the concentration of anaesthetic required for maintenance varies with the weight of the patient; that the dials of a McKesson machine measure the volume of gases flowing; that the Oxford vaporizer delivers warm ether; and that the main function of the expiratory valve of "a. Boyle" 